\title{
Dosimetric impact of bladder filling on organs at risk with barium contrast in the small bowel for adjuvant vaginal cuff brachytherapy
}

\author{
Maria del Carmen Salas Buzón, PhD', Lucia Gutiérrez Bayard, PhD', Raquel Rodriguez Sanchez, MD!', \\ Luis Ángel Quiñones Rodriguez, MD², Sarah Sayago Gil, MDl, Cristina Muñoz Higueras, MD' \\ IRadiation Oncology Department, Puerta del Mar University Hospital, Cádiz, Spain, ${ }^{2}$ Radiophysics Department, Puerta del Mar University \\ Hospital, Cádiz, Spain
}

\begin{abstract}
Purpose: The aim of this prospective study was to analyze dosimetric impact of modifying bladder filling on dose distribution in organs at risk (OARs) when using contrast in the small bowel of patients under adjuvant therapy with high-dose-rate vaginal cuff brachytherapy (HDR-VCB) for endometrial cancer.

Material and methods: This research included 19 patients who underwent laparoscopic surgery. They were treated with HDR-VCB and 2.5-3.5 cm diameter cylinders. Two successive computerized tomography (CT) scans were performed, with empty bladder and with bladder filled with $180 \mathrm{cc}$ of saline solution. Bladder, rectum, sigmoid, and small bowel were delineated as OARs. Oral barium contrast was used to clearly visualize small bowel loops. Prescription dose was 7 Gy. Dose-volume histograms were generated for each OAR, with full and empty bladder to compare doses received.

Results: Bladder distension had no dosimetric impact on the bladder, rectum, or sigmoid, unlike the small bowel. With full bladder, mean minimum dose at $2 \mathrm{cc}\left(\mathrm{D}_{2 \mathrm{cc}}\right)$ was not significantly higher for full vs. empty bladder (5.56 vs. $5.06 \mathrm{~Gy}, p=0.07)$, whereas there was a significant reduction in the small bowel (1.68 vs. $2.70 \mathrm{~Gy}, p<0.001)$. With full bladder, the dose increased to $50 \%$ of the volume $\left(\mathrm{D}_{50 \%}\right)$ of the bladder $(2.11 \mathrm{vs} .1 .28 \mathrm{~Gy}, p<0.001)$, and decreased in the small bowel ( 0.70 vs. $1.09 \mathrm{~Gy}, p<0.001)$.

Conclusions: The present study describes the dose received by organs at risk during HDR-VCB, making it possible to define the dose received by small bowel loops, when visualized with oral barium contrast. In patients undergoing laparoscopic surgery, a full bladder during HDR-VCB reduces the dose to the small bowel without a clinically relevant dose increase in the bladder, and no dose increase in other OARs.
\end{abstract}

J Contemp Brachytherapy 2021; 13, 6: 655-662 DOI: https://doi.org/10.5114/jcb.2021.112117

Key words: endometrial cancer, vaginal cuff brachytherapy, small bowel, bladder distention, barium.

\section{Purpose}

Endometrial carcinoma is one of the most common gynecologic tumors in the world [1]. Post-operative highdose-rate brachytherapy with vaginal cylinder to irradiate the vaginal cuff (HDR-VCB) has established itself as adjuvant radiotherapy treatment of early endometrial cancer [2-4].

The goal of HDR-VCB is to reduce local recurrence rates in vaginal cuff, but it may increase normal tissue toxicity [5]. Therefore, it is important to measure the dose received by normal tissues and pelvic organs at risk (OARs) during treatment [6, 7].

Laparoscopic approach to routine procedure for endometrial cancer modifies the technique of suturing the vaginal stump, which can be performed transvaginally [8]. There is a possibility that small bowel loops may remain very close to, and even attached to the inner end of vaginal cuff. Higher doses may be received in HDR-VCB treatment due to the proximity of vaginal cylinder, which cause unwanted intestinal morbidity [9].

Currently, treatment planning with HDR-VCB is based on the use of CT after gynecologic application [10,11]. In delineation of clinical target volume (CTV), the American Brachytherapy Society (ABS) and GEC-ESTRO (the Groupe Européen de Curiethérapie and the European Society for Radiotherapy and Oncology) recommendations are followed [12, 13]. Clinical target volume, which is considered the upper third portion of the vagina, is usually between $3 \mathrm{~cm}$ and $5 \mathrm{~cm}$ in length, with longer length
Address for correspondence: María del Carmen Salas Buzón, PhD, Radiation Oncologist, Radiation Oncology Department, Puerta del Mar University Hospital, Avenida Alcalde Álvaro Domecq 39, Casa “El sueño", Jerez de la Frontera, CP:11407, Cádiz, España, phone: 34-626990555,

•-mail: mcsalasbuzon@gmail.com
Received: 18.04 .2021

Accepted: 26.09 .2021

Published: 30.12 .2021 
in cases of adverse histology $[6,14,15]$. The bladder [16], rectum, sigmoid colon [13], and small bowel [13, 17] should be deemed as organs at risk within the pelvis. In current guidelines, there are no recommendations for the use of oral contrast in the small bowel during contouring planning [13]. After laparoscopic surgery, it would help to distinguish small bowel loops from the sigmoid and rectum. Regarding the rectum, enema before cylinder insertion does not influence recto-sigmoid dosimetric parameters [18].

\section{Non-consensus for HDR-VCB}

There is no consensus on several specific aspects when administering HDR-VCB treatment, such as bladder filling [19], placement of Foley catheter in the bladder [20, 21], amount of volume to fill the bladder [19, 21, 22], and procedure for a precise delimitation of the small bowel, the most radiosensitive organ relative to the bladder, rectum, and sigmoid $[19,23]$. In patients undergoing laparoscopic surgery and HDR-VCB, administration of oral barium contrast offers the advantage of clearly defining small bowel loops proximal to the vaginal cuff to differentiate them from other bowel loops in order to evaluate dosimetric consequences in this OAR with different bladder fill-

Table 1. Patients' characteristics

\begin{tabular}{|c|c|}
\hline Characteristics & No. of patients \\
\hline \multicolumn{2}{|l|}{ Age (years) } \\
\hline$\leq 60$ & 6 \\
\hline$>60$ & 13 \\
\hline \multicolumn{2}{|l|}{ Histology } \\
\hline Endometrioid & 16 \\
\hline Serous & 3 \\
\hline \multicolumn{2}{|l|}{ Tumor grade } \\
\hline G1 & 11 \\
\hline $\mathrm{G} 2$ & 4 \\
\hline G3 & 4 \\
\hline \multicolumn{2}{|l|}{ Surgery } \\
\hline $\mathrm{TLH}+\mathrm{BSO}$ & 6 \\
\hline $\mathrm{TLH}+\mathrm{BSO}+\mathrm{LNP}$ & 10 \\
\hline $\mathrm{TLH}+\mathrm{BSO}+\mathrm{LNP}+\mathrm{LNPR}$ & 3 \\
\hline \multicolumn{2}{|l|}{ Stage } \\
\hline IA & 6 \\
\hline IB & 13 \\
\hline \multicolumn{2}{|l|}{ Cylinder diameter (cm) } \\
\hline 2.5 & 4 \\
\hline 3.0 & 9 \\
\hline 3.5 & 6 \\
\hline \multicolumn{2}{|l|}{ Length treatment $(\mathrm{cm})$} \\
\hline 3 & 12 \\
\hline$>3$ & 7 \\
\hline Median dose (Gy) & 7 \\
\hline
\end{tabular}

ing. There are no published studies that assess dosimetric impact on OARs by modifying bladder filling in HDRVCB treatment in laparoscopic surgery and oral barium contrast in the small bowel. Therefore, this was the aim of the present study.

It is worth highlighting that a novel aspect of this study is the use of oral barium contrast in the small bowel during CT treatment planning in HDR-VCB.

\section{Material and methods}

\section{Patients}

Between November 2019 and December 2020, 19 women were included in this prospective dosimetric study. Prior approval was obtained from the Hospital's ethics committee. None of the patients had previously received HDR-VCB or external pelvic radiotherapy. Inclusion criteria were total laparoscopic hysterectomy (TLH) plus bilateral salpingo-oophorectomy and pelvic \pm para-aortic lymphadenectomy, confirmed histology of endometrial carcinoma with no other cancer, age over 18 years, and good performance status. Each patient provided written consent to participate in the study.

Staging of the International Federation of Gynecology and Obstetrics (FIGO) 2009 and pathologic characteristics of the patients are shown in Table 1.

\section{Treatment preparation and planning}

All the patients received exclusively vaginal cuff HDR-VCB. Three hours before the procedure, oral barium contrast and rectal micro-enema were applied. At baseline, a detailed gynecologic examination was performed to evaluate the vaginal cuff and select the diameter of cylinder. A Foley catheter was then inserted with $7 \mathrm{ml}$ of saline solution. Intracavitary HDR-VCB was performed with a magnetic resonance imaging (MRI), compatible plastic applicator with cylinder diameters between 2.5 and $3.5 \mathrm{~cm}$. The applicator was fixed with CT/ MRI applicator clamp (Elekta Company) with the cylinder positioned horizontally and legs extended, maintained throughout the treatment.

On the first day of treatment, two scans were performed successively, keeping the patient immobile in the same position: first scan with the bladder emptied and the second one with the bladder filled with $180 \mathrm{cc}$ of saline solution and $5 \mathrm{cc}$ of iodinated contrast, using a clamp holding the catheter. We used contrast in the bladder, following the same institutional protocol used in gynecological brachytherapy. The pelvis was scanned from the lumbar region to the ischial tuberosity. CT images were acquired with Siemens Somatom Confidence RT Pro scanner, with $120 \mathrm{KV}$ and $1.5 \mathrm{~mm}$ thick slices.

Both sets of CT images were transferred to Oncentra ${ }^{\circledR}$ Brachy comprehensive treatment planning for brachytherapy system (Elekta Company, Veenendaal, The Netherlands). Vaginal length was measured on CT images.

The external contour of cylinder was delimited in the desired treatment length of $3-5 \mathrm{~cm}$. The cylinder has not been excluded from CTV, but this did not influence the study, as the way the treatment was reproduced in empty 
and full bladder planning, making CTVs dose distributions identical.

A 5-mm expansion over the cylinder contour was performed automatically in all axes, except for the lower longitudinal, forming CTV. The dose of 7 Gy to CTV was prescribed for $3 \mathrm{~cm}$ and $3.5 \mathrm{~cm}$ cylinders, and 6.4 Gy for $2.5 \mathrm{~cm}$ cylinder, to administer the same equivalent dose $\left(\mathrm{EQD}_{2}\right)$ to the cylinder surface. One fraction per week was administered. Total administered doses were 21.0 Gy and 19.2 Gy, respectively. Dose calculation in OARs was performed following ICRU 89 standards [24].

Plans were performed with Oncentra Brachy v. 4.5.3 planning system. First, the planning was carried out on empty bladder CT scan. For this purpose, dose points were generated at $0.5 \mathrm{~cm}$ from the cylinder surface, following CTV length. Then, the plan dose was optimized and prescribed, so that $100 \%$ doses were uniform at these points for source dwells positions set to $0.25 \mathrm{~cm}$. Later, dwell positions and times obtained in empty bladder planning were reproduced on full bladder CT. Calculation algorithm was based on a TG43 formalism, which is the established standard for brachytherapy calculations, and does not consider density differences.

Finally, the treatment was delivered using an ${ }^{192} \mathrm{Ir}$ gamma radiation source using Nucletron MicroSelectron Afterloader (Elekta Instrument AB; Stockholm, Sweden).

The dosimetric values were expressed as the minimum dose at $0.1 \mathrm{cc}, 1.0 \mathrm{cc}$, and $2.0 \mathrm{cc}$ volume $\left(\mathrm{D}_{0.1 \mathrm{cc}}\right.$ to $\mathrm{D}_{2 \mathrm{cc}}$ ) plus exposed OAR, and the dose received by $50 \%$ of OAR volume $\left(\mathrm{D}_{50 \%}\right)$. For OAR volumes receiving $20 \%$ and $50 \%$ of the prescribed dose $\left(\mathrm{V}_{20}\right.$ and $\mathrm{V}_{50}$ in cc), the dose was delivered at $5 \mathrm{~mm}$ from the cylinder surface. The prescribed dose was administered in the first fraction with the bladder previously emptied by Foley catheter, and in the two successive fractions with the bladder empty, without Foley catheter. The rest of sessions were conducted with an empty bladder, with the same dosimetry as set on the first day. Patients not included in this study were treated with an empty bladder using the same dosimetric protocol.

\section{OARs contouring}

The bladder, rectum, sigmoid, and small bowel were considered OARs, and were contoured as solid organs in 3 dimensions by one physician and checked by another. They were contoured on CT with empty bladder and with full bladder. The rectum was contoured from the anal sphincter to the sigmoid colon area, where the rectum loses its' rounded shape in the axial plane and adopts a vertical orientation. It stops when the colon ascends laterally. The small bowel was contoured using barium contrast as an aid, up to $4 \mathrm{~cm}$ above the upper edge of the cylinder.

\section{Statistics}

The volumes of all specified OARs were determined, and dose-volume histograms generated in empty and full bladder states were compared. We planned the study with a continuous response variable from matched pairs.
Prior data [21] indicated that the difference in the response of bladder $\mathrm{D}_{2 \mathrm{cc}}$ dose pairs is normally distributed, with a standard deviation of $1.22 \mathrm{~Gy}$. According to Guler et al., for a true difference in the mean response of $0.85 \mathrm{~Gy}$ in the bladder $\mathrm{D}_{2 \mathrm{cc}}$, we would need to study 19 patients for a significance level of 0.05 and statistical power of 0.8. Statistical evaluation was performed on dosimetric changes produced by anatomical changes, and all statistical analysis were assessed with SPSS software, version 21.0 (SPSS, Chicago, IL, USA). Graphics were made with Microsoft Excel $365^{\circledR}$.

\section{Results}

The mean patient age of the 19 analyzed patients was 58 years (range, 38-81 years). Patients' characteristics, including FIGO stage (2017), histology, tumor grade, surgery, cylinder size, and dose per fraction are shown in Table 1. The number of fractions treated was 3 in all patients. None of the patients experienced discomfort due to Foley catheter or cylinder insertion. The mean full bladder volume was approximately four times larger compared to empty bladder $(248.54 \mathrm{cc}$ vs. $63.77 \mathrm{cc}$, $p<0.001$ ). With an empty bladder, the small bowel generally moved anteriorly, laterally, and superiorly, as presented in Figure 1.

Bladder distension did not have a significant dosimetric impact on the bladder with $\mathrm{D}_{2 \mathrm{cc}}$ from 5.06 to $5.56 \mathrm{~Gy}$ $(p=0.07)$, nor on the rectum or sigmoid, but it did have a significant impact on the small bowel, with $\mathrm{D}_{2 \mathrm{cc}}$ from 2.70 Gy to 1.68 Gy $(p<0.001)$ and $D_{50 \%}$ from 1.09 to $0.70 \mathrm{~Gy}$ with a full bladder (Table 2). $\mathrm{D}_{50 \%}$ of the bladder was affected by bladder distension from 1.28 to $2.11 \mathrm{~Gy}$, $p<0.001$. In Figure 2, $\mathrm{D}_{2 \mathrm{cc}}$ and $\mathrm{D}_{50 \%}$ values for each individual patient are displayed the for bladder in Figure $2 \mathrm{~A}$ and $\mathrm{B}$, and for the small bowel in Figure $2 \mathrm{C}$ and $\mathrm{D}$.

\section{Discussion}

In our study, the dosimetric effect of bladder filling in HDR-BT treatment with distended bladder significantly reduced the highest dose in the small bowel proximal to the vaginal cuff, visualized with oral barium contrast, with no significant change in the bladder, rectum, or sigmoid dose. To our knowledge, this is the first prospective study using barium contrast for small bowel delineation in post-operative HDR-BT treatment of vaginal cuff in patients operated by laparoscopy, facilitating differentiation with other bowel loops.

There are few studies [19-23] comparing dosimetric influence of bladder filling during HDR-VCB planning in OARs, and there are no studies quantifying a difference between using or not using contrast in the small bowel. All published studies are presented in Table 3.

Hoskin and Vidler are the first authors to perform a comparative analysis of empty and full bladder. They based their measurements on a 2D bladder height, without evaluating volumetric parameters of the bladder. They found that instillation of $100 \mathrm{ml}$ of water into the bladder decreased the amount of small bowel in highdose region by $57 \%$, without significantly increasing the 

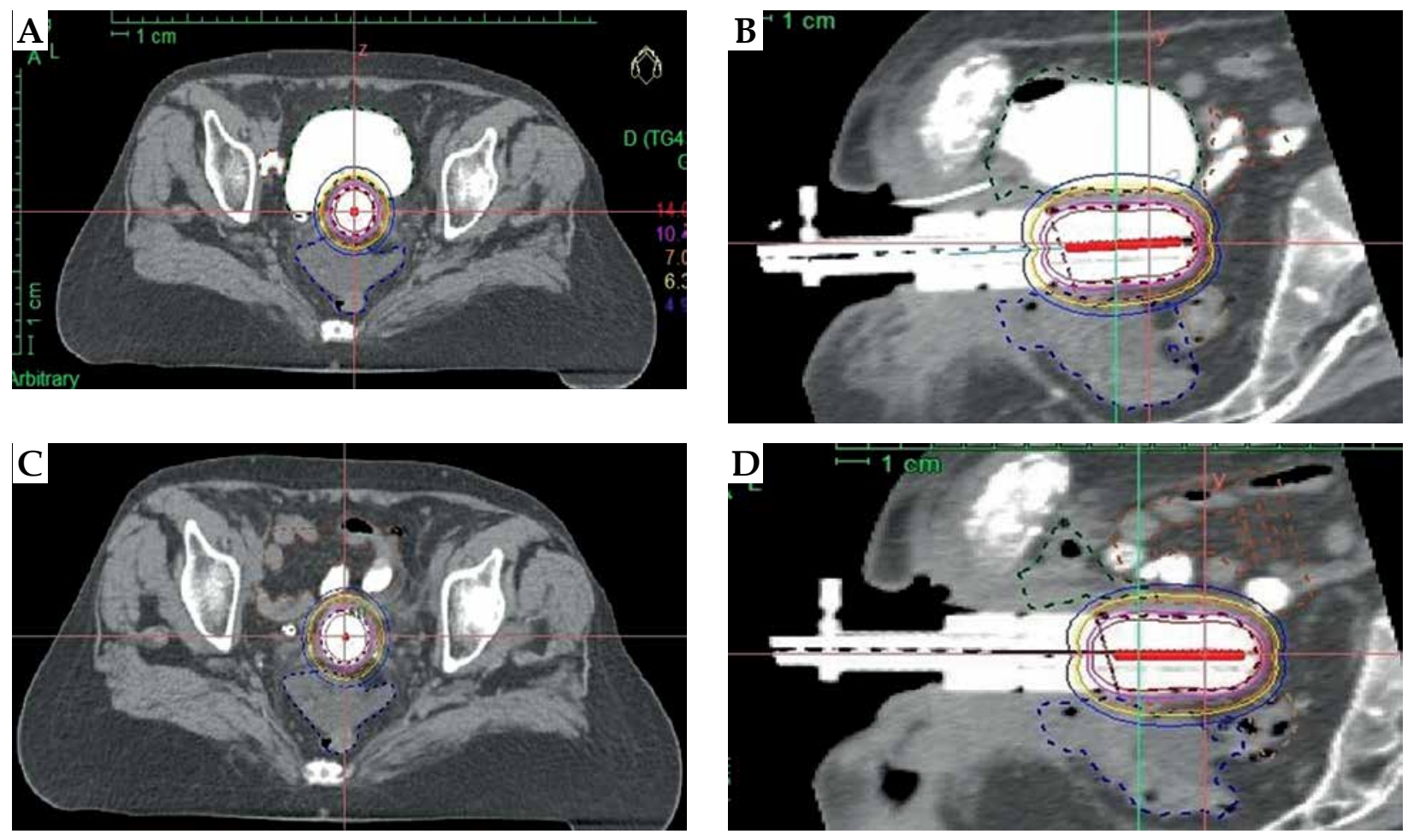

Fig. 1. Organs at risk (OARs) in axial with full bladder (A) and sagittal with full bladder (B) (small bowel with barium contrast displaced anteriorly, superiorly, and laterally); in axial with empty bladder (C) and sagittal direction with empty bladder (D) (small bowel loops with barium contrast located very close to the cylinder)

Table 2. Mean dosimetric values of bladder and small bowel in full and empty bladder filling

\begin{tabular}{lccc} 
& \multicolumn{2}{c}{ Mean dosimetric values } & \multirow{2}{*}{$p$-value } \\
\cline { 2 - 3 } & \multicolumn{1}{c}{ Empty bladder } & Full bladder & $<0.001$ \\
\hline Mean volume bladder (range), cc & $62.18(25.05-112.3)$ & $248.0(156.5-307.0)$ & $<0.05$ \\
\hline Mean volume small bowel (range), cc & $63.48(26.2-131.7)$ & $40.63(23.9-174.4)$ & $<0.001$ \\
\hline$D_{50 \%}$ for bladder (range), Gy & $2.11(1.03-3.63)$ & $1.28(0.63-2.18)$ & $<0.001$ \\
\hline$D_{50 \%}$ for small bowel (range), Gy & $1.09(0.18-1.78)$ & $0.70(0.24-1.8)$ & 0.15 \\
\hline$D_{0.1 c c}$ for bladder (range), Gy & $6.60(4.4-8.6)$ & $7.05(3.2-9.47)$ & $<0.05$ \\
\hline$D_{0.1 c c}$ for small bowel (range), Gy & $3.90(0.34-8.64)$ & $2.61(0.97-11.14)$ & $<0.05$ \\
\hline$D_{1 c c}$ for bladder (range), Gy & $5.54(3.4-7.3)$ & $6.18(4-7.71)$ & $<0.001$ \\
\hline$D_{1 c c}$ for small bowel (range), Gy & $3.07(0.5-7.8)$ & $1.97(0.23-4.26)$ & 0.07 \\
\hline$D_{2 c c}$ for bladder (range), Gy & $5.06(2.9-6.66)$ & $5.56(2.5-7.36)$ & $<0.001$ \\
\hline$D_{2 c c}$ for small bowel (range), Gy & $2.70(0.5-4.81)$ & $1.68(0.22-4.06)$ & $<0.001$ \\
\hline$V_{20}$ for bladder (range), \% & $74.13(24.23-99.24)$ & $44.97(9.13-83.4)$ & $<0.001$ \\
\hline$V_{20}$ for small bowel (range), \% & $28.98(0.0-84.6)$ & $13.75(0-71.41)$ & $<0.001$ \\
\hline$V_{50}$ for bladder (range), \% & $18.40(1.13-53.89)$ & $8.90(1.16-16.43)$ & 0.14
\end{tabular}

$D_{0.1 c c}$-dose to $0.1 c c ; D_{1 c c}$-dose to $1 c c ; D_{2 c c}$-dose to $2 c c ; D_{50 \%}$-dose to $50 \%$ of volume; $V_{20 \%}$ - percentage of organ volume receiving $\geq 20 \%$ of the prescribed dose; $V_{50 \%}$ - percentage of organ volume receiving $\geq 50 \%$ of the prescribed dose

mean maximum bladder dose. Full bladder treatment with at least $100 \mathrm{cc}$ is recommended [22].

Steward et al. investigated dose parameters in CT planning with empty and full bladder on images acquired 1 hour after consumption of $32 \mathrm{oz}$ of water. They reported that bladder distension increased bladder $\mathrm{D}_{2 \mathrm{cc}}$, with no appreciable change in rectal $\mathrm{D}_{2 \mathrm{cc}}$. Sigmoid colon and small bowel dosimetrics were not evaluated. In contrast, our result showed that bladder $\mathrm{D}_{2 \mathrm{cc}}$ was $+10 \%$ change, but not significantly higher with a full bladder. As the small bowel was not considered, we could not compare data in this OAR [19]. 
A

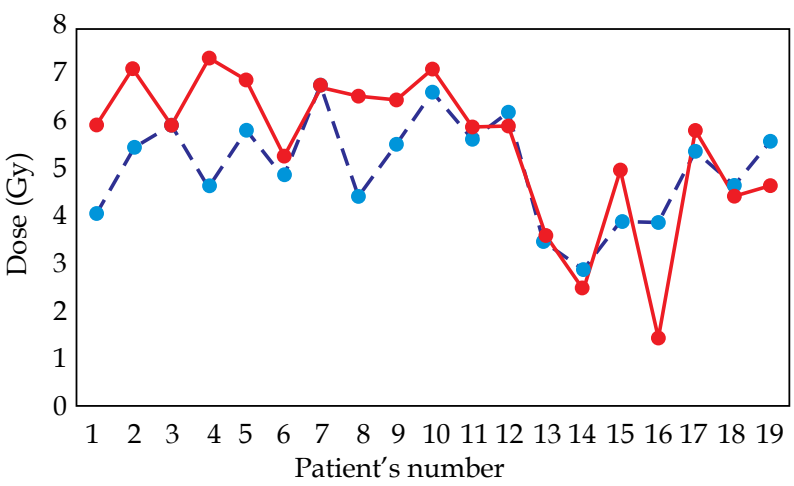

C

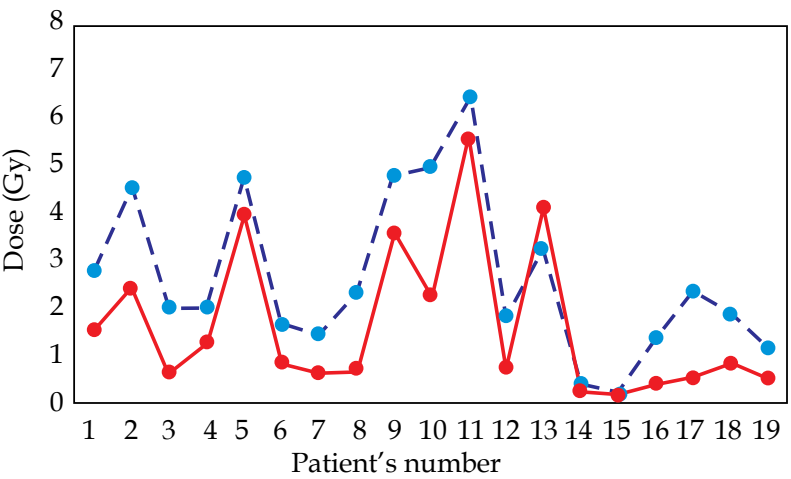

B



D

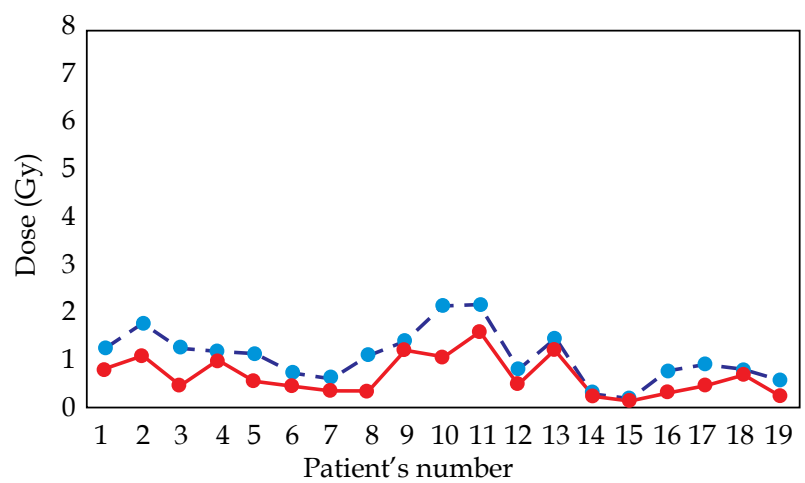

Fig. 2. Values $D_{2 c c}(G y)$ and $D_{50 \%}$ for individual case. Dashed line: empty bladder, red line: full bladder. A) $D_{2 c c}$ bladder; B) $\mathrm{D}_{50 \mathrm{cc}}$ bladder; C) $\mathrm{D}_{2 \mathrm{cc}}$ small bowel; D) $\mathrm{D}_{50 \mathrm{cc}}$ small bowel

Hung et al. evaluated OARs doses to the rectum, bladder, sigmoid, and small bowel without a contrast. $\mathrm{D}_{2 \mathrm{cc}}$ (1.179 cGy vs. 1.246 cGy, $p=0.11$ ) and $\mathrm{D}_{50 \%}$ (441 cGy vs. $279 \mathrm{cGy}, p=0.001$ ) of small bowel with bladder filled with $180 \mathrm{cc}$ via Foley catheter, resulted in a significant dose reduction with no significant changes in the bladder, rectum, or sigmoid. Our dosimetric calculation is in agreement with these findings, although the dose recorded at 2 cc bladder with full bladder was slightly higher, it was not significant. In addition, the dose to the small bowel was significantly lower with a full bladder. Their study was performed without oral contrast in small bowel. The authors advised treatment with distended bladder [23].

In a study by Kobzda et al., the bladder was filled with a $400 \mathrm{ml}$, ingested $40 \mathrm{~min}$ before CT. They described that bladder $\mathrm{D}_{2 \mathrm{cc}}$ significantly decreased with empty bladder (4.9 Gy vs. 4.6 Gy, $p<0.05$ ), while volumetric doses $\left(\mathrm{V}_{50}\right.$ and $\left.\mathrm{V}_{80}\right)$ were significantly higher in the empty organ. Our results show a similar trend, but without statistical significance. This difference could be explained by a different volume of bladder filling used (400 cc vs. $180 \mathrm{cc}$ ). In the small bowel, it was associated with a significant reduction of the intestinal dose of $\mathrm{D}_{2 \mathrm{cc}}(4.1 \mathrm{~Gy}$ vs. $4.6 \mathrm{~Gy}, p<0.05)$. Their findings are consistent with the present results. Moreover, the authors recommended treatment with a full bladder [20].

In a study, Guler $e$ t al. assessed patients who underwent initial CT with an empty bladder and then, sterile saline $(180 \mathrm{ml})$ was infused through a Foley catheter. Two-thirds of the vaginal cuff was treated. OARs, such as the bladder, rectum, sigmoid, and small bowel without contrast were analyzed. Full bladder produced a significant $18.7 \%$ increase in $\mathrm{D}_{2 \mathrm{cc}}$ in the bladder (5.40 Gy vs. $4.55 \mathrm{~Gy}, p<0.05$ ), but not in the rectum. The reduction in sigmoid and small bowel was not significant. The results of our study are not consistent with these findings. One cause could be that their treatment activated two thirds of the vaginal cylinder, while in our protocol, only one third was activated. The other also did not use contrast in the small bowel [21].

Our dosimetric study has some limitations, including small number of cases, which makes it difficult to generalize the results. This was a dosimetric study that did not intend to draw clinical conclusions. The obtained data should be correlated with short- and long-term morbidity analyses, quality of life questionnaires, and in vivo dosimetry studies. Such a correlation could help to assess the doses to OARs, suggesting further research development.

\section{Conclusions}

In patients undergoing laparoscopic surgery for endometrial cancer treated with HDR-VCB, full bladder reduces a higher dose to the small bowel previously visualized with oral barium contrast, without a clinically relevant dose increase in the bladder and no dose increase in the sigmoid or rectum. Treatment with full bladder appears more beneficial dosimetrically due to possible impact on this risk organ. 


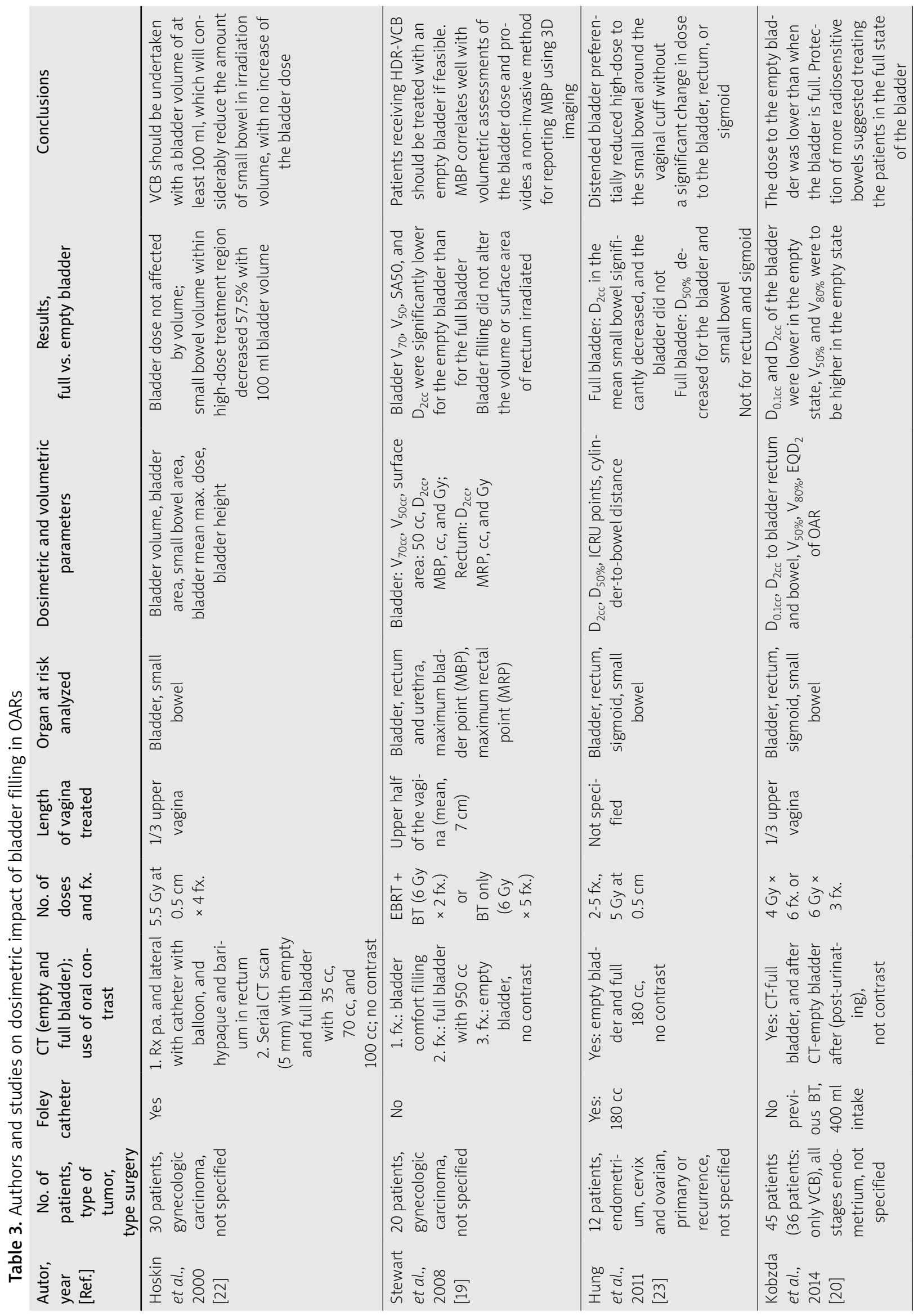




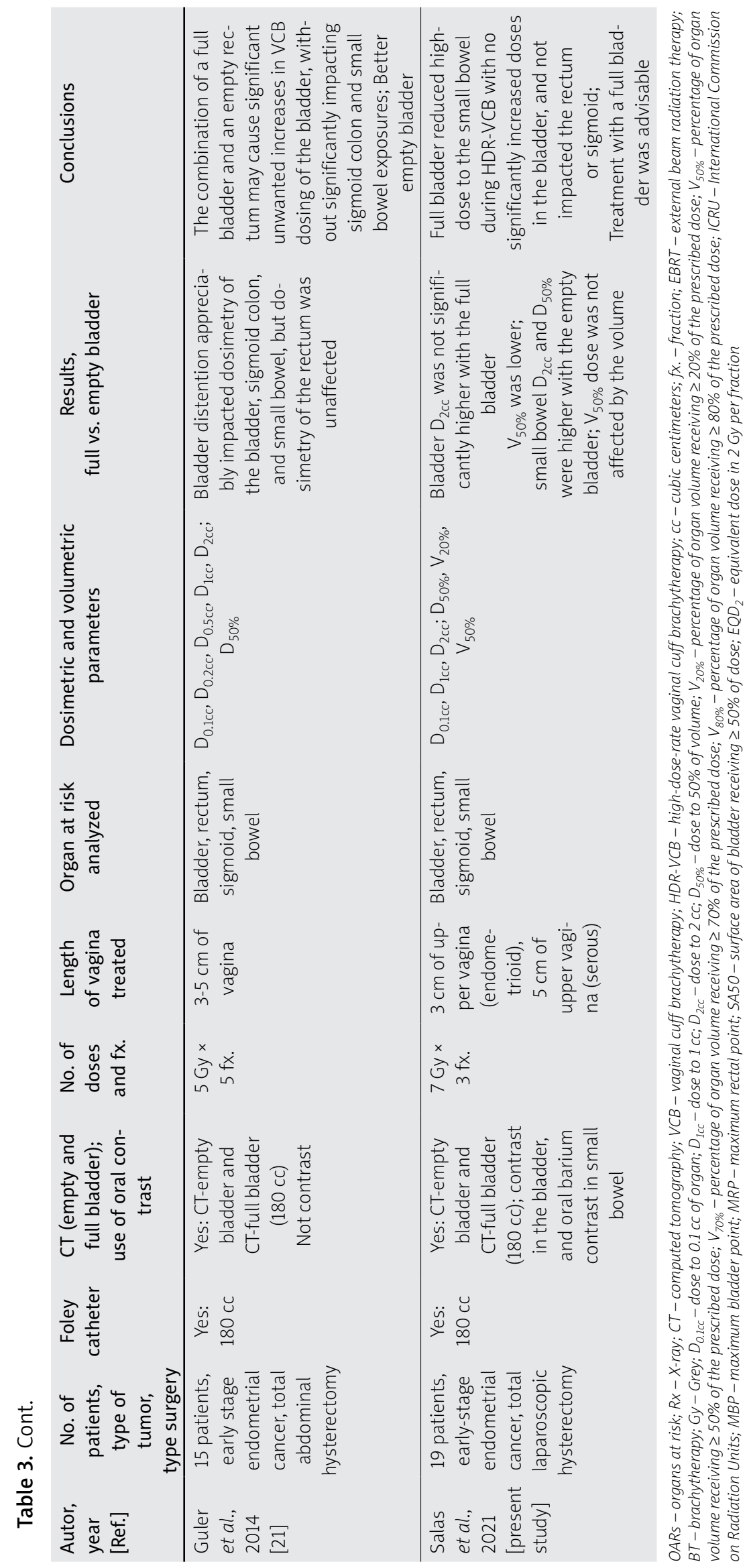




\section{Disclosure}

The authors report no conflict of interest.

\section{References}

1. Sung H, Ferlay J, Siegel RL et al. Global Cancer Statistics 2020: GLOBOCAN estimates of incidence and mortality worldwide for 36 cancers in 185 countries. CA Cancer J Clin 2021; 71: 209-249.

2. Colombo N, Creutzberg C, Amant F et al., ESMO-ESGOESTRO Endometrial Consensus Conference Working Group. ESMO-ESGO-ESTRO Consensus Conference on Endometrial Cancer: diagnosis, treatment and follow-up. Ann Oncol 2016; 27: 16-41.

3. Concin N, Matias-Guiu X, Vergote I et al. ESGO/ESTRO/ ESP guidelines for the management of patients with endometrial carcinoma. Int J Gynecol Cancer 2021; 31: 12-39.

4. Serkies K, Baczkowska-Waliszewska Z. Adjuvant vaginal cuff brachytherapy in surgically treated endometrial carcinoma patients - in view of the recent evidence. J Contemp Brachytherapy 2021; 13: 221-230.

5. Autorino R, Tagliaferri L, Campitelli M et al. EROS study: Evaluation between high-dose-rate and low-dose-rate vaginal interventional radiotherapy (brachytherapy) in terms of overall survival and rate of stenosis. J Contemp Brachytherapy 2018; 10: 315-320.

6. Small W, Beriwal S, Demanes DJ et al. American Brachytherapy Society consensus guidelines for adjuvant vaginal cuff brachytherapy after hysterectomy. Brachytherapy 2012; 11: 58-67.

7. Sabater S, Andres I, Lopez-Honrubia V et al. Vaginal cuff brachytherapy in endometrial cancer - a technically easy treatment? Cancer Manag Res 2017; 9: 351-362.

8. Uccella S, Malzoni M, Cromi A et al. Laparoscopic vs transvaginal cuff closure after total laparoscopic hysterectomy: a randomized trial by the Italian Society of Gynecologic Endoscopy. Am J Obstet Gynecol 2018; 218: 500.e1-500.e13.

9. Caon J, Holloway C, Dubash R et al. Evaluating adjacent organ radiation doses from postoperative intracavitary vaginal vault brachytherapy for endometrial cancer. Brachytherapy 2014; 13: 94-99.

10. Harkenrider MM, Grover S, Erickson BA et al. Vaginal brachytherapy for postoperative endometrial cancer: 2014 Survey of the American Brachytherapy Society. Brachytherapy 2016; 15: 23-29.

11. Gultekin M, Yilmaz MT, Biltekin F et al. Adjuvant vaginal cuff brachytherapy: dosimetric comparison of conventional versus 3-dimensional planning in endometrial cancer. J Contemp Brachytherapy 2021; 12: 601-605.

12. Small W, Erickson B, Kwakwa F. American Brachytherapy Society survey regarding practice patterns of postoperative irradiation for endometrial cancer: Current status of vaginal brachytherapy. Int J Radiat Oncol Biol Phys 2005; 63: 1502 1507.

13. Hoskin P, Hellebust TP, Nout R et al. Endometrial cancer. In: van Limbergen E, R. Potter, P. Hoskin (Eds.). The GEC ESTRO Handbook of Brachytherapy. Brussels, December 2019.

14. Eldredge-Hindy HB, Eastwick G, Anne PR et al. Adjuvant vaginal cuff brachytherapy for high-risk, early stage endometrial cancer. J Contemp Brachytherapy 2014; 6: 262-270.

15. Harkenrider MM, Block AM, Alektiar KM et al. American Brachytherapy Task Group Report: Adjuvant vaginal brachytherapy for early-stage endometrial cancer: A comprehensive review. Brachytherapy 2017; 16: 95-108.

16. Nag S, Erickson B, Parikh S et al. The American Brachytherapy Society recommendations for high-dose-rate brachythera- py for carcinoma of the endometrium. Int J Radiat Oncol Biol Phys 2000; 48: 779-790.

17. Viswanathan AN, Erickson BA. Three-dimensional imaging in gynecologic brachytherapy: a survey of the American Brachytherapy Society. Int J Radiat Oncol Biol Phys 2010; 76: 104-109.

18. Sikorska K, Zolciak-Siwinska A, Kowalczyk A et al. Dosimetric evaluation of vaginal cuff brachytherapy planning in cervical and endometrial cancer patients. J Contemp Brachytherapy 2020; 12: 248-251.

19. Stewart AJ, Cormack RA, Lee $\mathrm{H}$ et al. Prospective clinical trial of bladder filling and three-dimensional dosimetry in high-dose-rate vaginal cuff brachytherapy. Int J Radiat Oncol Biol Phys 2008; 72: 843-848.

20. Kobzda JD, Cikowska-Wozniak E, Michalska $M$ et al. Three-dimensional dosimetry of the full and empty bladder in high dose rate vaginal cuff brachytherapy. Int J Gynecol Cancer 2014; 24: 923-927.

21. Guler OC, Onal C, Acibuci I. Effects of bladder distension on dose distribution of vaginal vault brachytherapy in patients with endometrial cancer. J Contemp Brachytherapy 2014; 6: 371-376.

22. Hoskin PJ, Vidler K. Vaginal vault brachytherapy: the effect of varying bladder volumes on normal tissue dosimetry. $\mathrm{Br}$ J Radiol 2000; 73: 864-866.

23. Hung J, Shen S, De Los Santos JF et al. Image-based 3D treatment planning for vaginal cylinder brachytherapy: Dosimetric effects of bladder filling on organs at risk. Int J Radiat Oncol Biol Phys 2012; 83: 980-985.

24. Prescribing, recording, and reporting brachytherapy for cancer of the cervix. J ICRU 2013; 13: NP. 